\title{
Lichen ruber mucosae der Mundschleimhaut
}

\author{
Enoral Lichen ruber mucosae
}

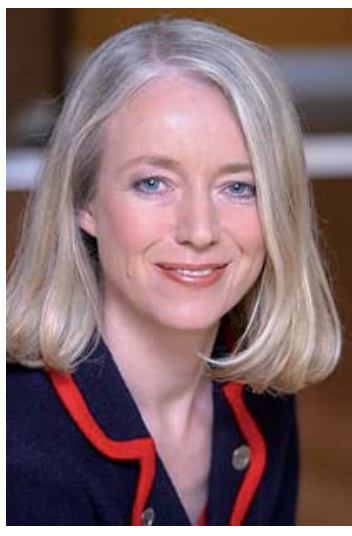

Prof. Dr. Christiane Bayerl
Der Lichen ruber planus mucosae der Mundschleimhaut ist eine chronische immunologische mukokutane entzündliche Erkrankung unbekannter Ätiologie. Neben Zunge und Wagenschleimhaut können auch Speiseröhre und Kehlkopf betroffen sein [1]. Vermutet werden zellvermittelte immunologische Prozesse bei Allergien, Autoimmunerkrankungen und Hepatitis-B- und -C-Infektionen. Weiter gilt der orale Lichen planus bei langem Bestehen als Präkanzerose, die unbedingt eine rechtzeitige Therapie fordert (siehe auch die Arbeit zu Lichen ruber planus verrucosus bei Vitiligo S. $425 \mathrm{ff}$ in diesem Heft). Die WHO hat den Lichen ruber mucosae als Präkanzerose klassifiziert [2]. Untersuchungen von 1924 bis 2007 zeigten eine maligne Transformation des oralen Lichen ruber bei $0-12,5 \%$, in großen Fallserien zwischen 1-2\%. Als Risiken werden Kortikosteroidtherapie, Hormonersatztherapie, HPV-Infektionen und das weibliche Geschlecht diskutiert [3].

Ich möchte Ihnen hier am Platz eines Editorials nicht die ganze Palette der Metaanalysen zu den Therapieoptionen darstellen. Eine Cochrane-Analyse zur Therapie des sehr schmerzhaften erosiven Lichen ruber planus der Mundschleimhaut zeigte aus 15 Studien, dass 0,025\% Clobetasolpropionat in flüssig Microspheren und in einer anderen Studie Ciclosporin-Lösung besser als 0,1\% Triamcinoloneacetonid in Orabase den Schmerz reduzierten. Schlechter schnitt Aloe-Vera-Gel ab, das nur 50\% Schmerzreduktion brachte. Die therapeutische Palette wird etwas breiter, wenn der Lichen ruber nicht erosiv ist, da dann auch Präparate eingesetzt werden können, die eine leichte Irritation auslösen können oder auf erosiven Stellen brennen würden wie topische Retinoide oder Calcineurinantagonisten $[2,4,5]$.

Ich möchte Sie mit zwei verblüffenden neuen Studien bekannt machen [6,7]. Verglichen wurde die intraläsionale Injektion von Triamcinolonacetonid versus Mundspülungen mit Triamcinolonacetonid bei 40 Probanden mit Lichen ruber mucosae. Beurteilungskriterien waren Schmerzen, Brennen und das Oral Health Impact Profile (OHEP-14), Rückfälle und Nebenwirkungen, evaluiert nach 6 Wochen. In beiden Gruppen waren Brennen und OHEP-14 gebessert und zwar gleich gut. Der visuelle analoge Schmerzscore (VAS) war in der Injektionsgruppe jedoch deutlich gesunken und Nebenwirkungen in der Mundspülgruppe höher, wie z. B. enorale Candida-Infektionen. Obwohl beide Therapien gleich effektiv waren, fielen die Nebenwirkungen in der Gruppe mit intraläsionaler Injektion deutlich geringer aus [6]. Dass Steroidinjektionen trotz der Injektion mit- tels einer Spritze besser „ankommen“ als Steroidspülungen bei Lichen ruber mucosae, verblüfft. Aufgrund der nicht ganz zufriedenstellenden Therapiesituation bei oralem Lichen ruber wurden Lichttherapieoptionen an der Mundschleimhaut erprobt. Die zweite für mich ungewöhnliche Studie hatte in einer kleinen Gruppe von 14 Patienten bei 9 Patienten ein komplettes Abheilen und bei 5 ein Teilansprechen auf eine UV-Therapie gezeigt. Verwendet wurde das TheraLight UV 120-2 System (Karlsbad, CA) mit kontinuierlicher Steigerung der UVB-Dosis (290-320 nm mit Betonung der Wellenlänge $304 \mathrm{~nm}$ über ein flexibles Fiberglas mit Öffnung von $1,9 \times 1,9 \mathrm{~cm}$ ) über 8 Wochen. 10 Patienten wurden sogar über 29 Wochen behandelt. Die Frequenz der Behandlungen betrug in den ersten 8 Wochen $3 \times$ pro Woche, Woche 9-12 zweimal pro Woche, und weiter ausschleichend über die Wochen. Immerhin bei einem Patienten wurde während der Studie eine dysplastische Läsion entfernt. 7\% der Patienten gaben Brennen an, sonst keine Nebenwirkungen [7]. Diese Therapieoption verblüfft, wurde doch in Hautmodellen ein potenzielles Risiko der UVBStrahlung für die Mundschleimhaut belegt mit 3D-Modellen menschlicher Haut (EpiDerm), für Gingiva (EpiGing) und orales Gewebe (EpiOral). Zytokine, initialer DNA-Schaden über Cyclobutan-Pyrimidin-Dimere (CPD), deren Reparatur und apoptotische Zellen wurden evaluiert. IL-8 und CPD waren erhöht, CPD-Reparaturraten und apoptotische Zellen waren zahlenmäßig geringer als in der Epidermis der verhornten Haut [8]. Komplettes Ansprechen wurde definiert als mindestens $80 \%$ und ein Teilansprechen als $50-80 \%$ Abheilung der erkrankten Mukosa, kein Ansprechen bei weniger als $50 \%$. Die Arbeit zeigt nur begrenzte Effekte und in keinem Fall komplette Abheilung. $\mathrm{Zu}$ folgern bleibt also, Hände weg von der Bestrahlung der Mundschleimhaut! Und auch ohne Bestrahlungstherapie gilt, halbjährliche Kontrollen eines Lichen ruber an Schleimhäuten - oral der genital - sind obligat.

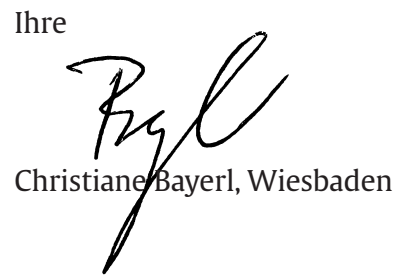

Die Literatur zu diesem Editorial finden Sie unter http://dx.doi.org/10.1055/s-0034-1393123. 